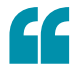

the cancer cells first attach and spread on top of the mesothelial cells, and then move underneath the mesothelial layer.

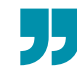

\section{OVARIAN CANCER}

\section{Driving force}

In order to metastasize, ovarian cancer cells form clusters (spheroids) that travel through the ascites fluid and attach to organs within the peritoneal cavity, a process that requires invasion of the mesothelial cell layer covering these organs. A lack of mesothelial cells directly under ovarian tumour spheroids has been observed in electron micrographs, but how this mesothelial clearance occurs is unknown. Joan Brugge and colleagues have begun to delve into the mechanisms underlying mesothelial clearance through the use of in vitro time-lapse video microscopy.

The authors observed the displacement of green fluorescent protein (GFP)-labelled lung mesothelial cells by spheroids that were formed from the ovarian cancer cell line OVCA433, as well as spheroids that were isolated from patients with ovarian cancer. OVCA433 spheroids also induced the clearance of mesothelial cells that were grown on a more physiologically relevant extracellular matrix (ECM), with a similar stiffness to connective tissue.

More detailed examination of the interactions between the two cell types revealed that the cancer cells first attach and spread on top of the mesothelial cells, and then move underneath the mesothelial layer. The authors hypothesized that the cancer cells were promoting the localized disruption of mesothelial cell-ECM adhesion, leading to movement of the mesothelial cells away from the cancer cells. Indeed, cancer spheroids induced matrix adhesion disassembly, as visualized by changes in GFP-paxillin (a protein that localizes to sites of integrin-ECM engagement) in mesothelial cells, and mesothelial cells that came into contact with a cancer spheroid migrated significantly further than those not in contact with a spheroid.

How do the cancer cells promote the de-adhesion and movement of mesothelial cells? Myosin contractility can be used by cells to exert force on the ECM, thus provoking adhesion changes in other nearby cells. Reducing the expression of myosin II isoforms in OVCA433 cells by RNA interference allowed adhesion to mesothelial cells but prevented mesothelial clearance. Exertion of force requires linkage of integrins such as a $5 \beta 1$ (a fibronectin receptor) to the actomyosin network through talin 1 (TLN1). Either small hairpin RNAs (shRNAs) against TLN1 or a blocking antibody against $\alpha 5$ integrin in OVCA433 spheroids prevented mesothelial clearance; similar data were obtained in spheroids that were formed from two other ovarian cancer cell lines. Furthermore, the overexpression of $\alpha 5$ integrin in ovarian cancer cells with low a 5 expression (OVCAR5) allowed mesothelial clearance. Imaging of fibronectin dynamics showed that OVCA433 spheroids can induce $\alpha 5 \beta 1$ integrin-dependent detachment of fibronectin fibrils from mesothelial cells, and traction force microscopy confirmed the importance of myosin, TLN1 and $\alpha 5 \beta 1$ integrin for OVCAR5 cells to generate force on a fibronectin substrate.

Overall, this suggests a model wherein $\alpha 5 \beta 1$ integrin on tumour cells interacts with fibronectin on mesothelial cells, leading to the activation of actomyosin contractility in the tumour cells. This results in the detachment of fibronectin from the mesothelial cells, allowing them to migrate away from the tumour cells. If these results are confirmed in vivo, it could explain how ovarian cancer cells penetrate the mesothelial barrier during metastasis.

Sarah Seton-Rogers

ORIGINAL RESEARCH PAPER Iwanicki, M. P. et al Ovarian cancer spheroids use myosin-generated force to clear the mesothelium. Cancer Discovery 14 Jun 2011 (doi:10.1158/2159-8274.CD-11-0010)

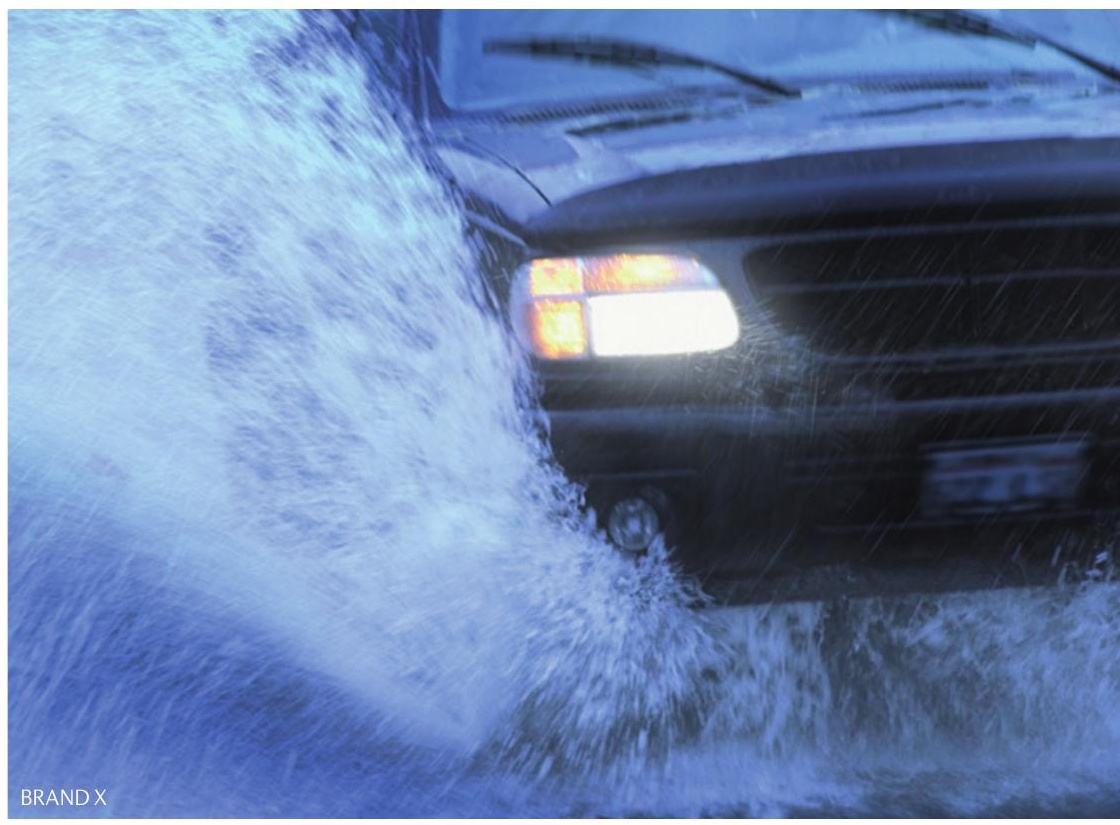

